\title{
Pattern Formation Using Metal Silver Staining on Cellulosic Substrate by Patterned Ultraviolet Light Irradiation
}

\author{
Hoon-sik Shin Yang-seob Moon, Seiji Tokino and Mitsuo Ueda \\ Department of Chemistry \& Materials Technology, Kyoto Institute of Technology, \\ Kaido-cho, Matsugasaki, Sakyo-ku, Kyoto-si, 606 Japan
}

\begin{abstract}
A simple image formation on cellulosic substrate with using a combination of patterned UV photooxidation reaction on cellulosic substrate and metal silver deposition reaction on the oxidized cellulose substrate has been studied. The color depth of the silver deposit on the substrate increased with increasing the integrated energy of UV irradiation. Assuming that the color depth of the deposit is proportional to the amount of aldehydes formed on the substrate, the photooxidation reaction of hydroxyl group on the cellulose substrate to aldehydes found to apparently follow the first order reaction kinetics.
\end{abstract}

\section{Introduction}

In the past several decades, various methods for the formation of images on polymer substrates or on papers have been developed, such as silver halides photography, dye printing, pigment printing, ink-jet printing, thermal transfer printing, electrophotography, etc. Elaborate chemicals or exquisitely designed mechanical or electronic devices, however, are necessary for these method to obtain fine images on the polymer substrate.

In this paper, we considered new method for the simple image formation on cellulosic substrate using ultraviolet light (UV) irradiation on the substrate and subsequent metal silver deposition by the reduction reaction from aqueous silver ion with aldehyde groups formed on the cellulosic substrate by the UV photooxidation.

It has been known that cellulosic substrate is easily photo-oxidized by UV irradiation forming di-aldehydes on the substrate $(1,2)$. Metal silver deposition caused by the reduction reaction with the aldehydes has also been well known and been practically utilized for one of the test method for the presence of oxy-cellulose on the cellulosic substrate, especially in the process for checking excess-bleaching of cellulosic substrate (3). Combining the UV photooxidation reaction on the cellulosic substrate and the metal silver deposition reaction on the oxidized cellulose substrate, a simple image on the cellulosic substrate can be produced by the dark micro-deposit staining with metal silver on the cellulosic substrate.

\section{Experiment}

Filter paper (Advantec No. 2; $5 \times 2 \mathrm{~cm}$ ) was used as a cellulosic substrate for measuring color depth of the metal silver deposition.

Low pressure mercury lamp (Sen Engineering Co. Ltd., SUV-40U) that radiates mainly 184.9 and $253.7 \mathrm{~nm}$ of UV light was employed as a UV light source at 4.5 $\mathrm{cm}$ distance from the substrates. Energy of the UV irradiation on the substrate was $0.375 \mathrm{~J} \mathrm{~min}^{-1} \mathrm{~cm}^{-2}$.

UV irradiated sample was rinsed in water for $1 \mathrm{~min}$ and was immersed in an aqueous solution $\left(85^{\circ} \mathrm{C}\right)$ that contains silver nitrate $(1 \% \mathrm{w} / \mathrm{w})$, sodium hydroxide $(4 \% \mathrm{w} / \mathrm{w})$ and sodium thiosulphate $(4 \% \mathrm{w} / \mathrm{w})$ for 10 min. The silver stained sample was then rinsed with water and dried.

Color depth of the sample was evaluated in $K / S$ value at $400 \mathrm{~nm}$ with a Minolta CM-1000 reflectance 
spectrophotometer. Apparent $K / S$ values were calculate from reflectivity on the fiber by KubelkaMunk equation.

\section{Results and Discussion}

$K / S$ value of the sample filter paper that was stained with micro-deposit of metal silver from aqueous silver ion solution after UV irradiation has been shown in Fig. 1 against time integrated energy of the UV irradiation. With increasing the time integrated energy of the UV irradiation on the filter paper, the color depth of the stained sample by the silver deposition increases. After $15 \mathrm{~J} \mathrm{~cm}^{-2}$ of the UV irradiation, the depth of the stained sample reaches plateau at ca. K/S 8.0. This indicates that UV photooxidation of the substrate, i.e.

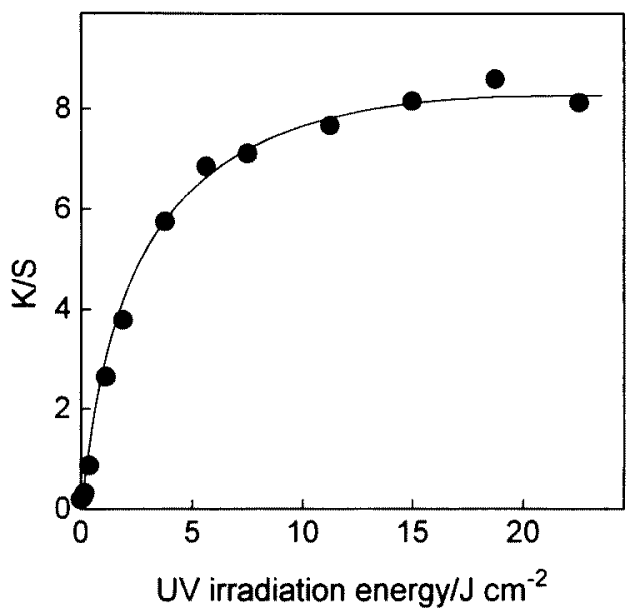

Fig. $1 K / S$ value of the sample filter paper that was stained with micro-deposit of metal silver from aqueous silver ion solution after UV irradiation.

conversion of the hydroxyl group of the cellulosic substrate to aldehydes, is responsible for the subsequent silver deposition and the yield of the conversion become plateau at $15 \mathrm{~J} \mathrm{~cm}^{-2}$ of the integrated energy of the irradiation. A supposed reaction scheme of the photo-chemical conversion of the cellulosic substrate by the UV irradiation is depicted in Fig. 2 [4]. This supposition would be supported by the fact (2) that aldehyde group on the UV irradiated cellulosic substrate has been detected with IR spectrophotometry. The supposition would also be supported by the fact

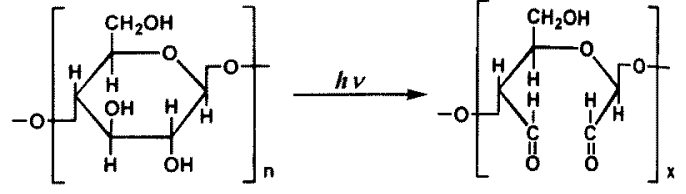

Fig. 2 Supposed reaction scheme of the photooxidation conversion of the cellulosic sub. strate by the UV irradiation (4).

that chemically oxidized cellulose with periodic acid that contains 2,3 di-aldehyde group $(2,5)$ in the cellulose chain showed strong reducing ability for aqueous silver ion forming dark metal silver deposit on the cellulose substrate [6].

Assuming that $K / S$ value of the UV irradiated and silver stained sample is proportional to the existence of the aldehydes on the cellulosic substrate, $\left[(K / S)_{*}\right.$ $\left.-(K / S)_{t}\right]$ would be proportional to the amount of the hydroxyl groups that is accessible to convert to aldehydes by the UV irradiation, where $(K / S)_{\infty}$ stands for the $K / S$ value of the sample that is UV irradiated with large enough energy correspond to the saturated aldehydes formation on the substrate.

An empirical linear relationship with logarithmic equation, as shown in Fig. 3, taking plots of $\log$ $\left[(K / S)_{\infty}-(K / S)_{t}\right]$ of the sample against the UV irradiation energy, where $(K / S)_{\infty}$ and $(K / S)_{t}$ stands for the $K / S$ values of the silver stained sample after the $\mathrm{UV}$ irradiation with large enough energy $\left(15 \mathrm{~J} \mathrm{~cm}^{-2}\right)$

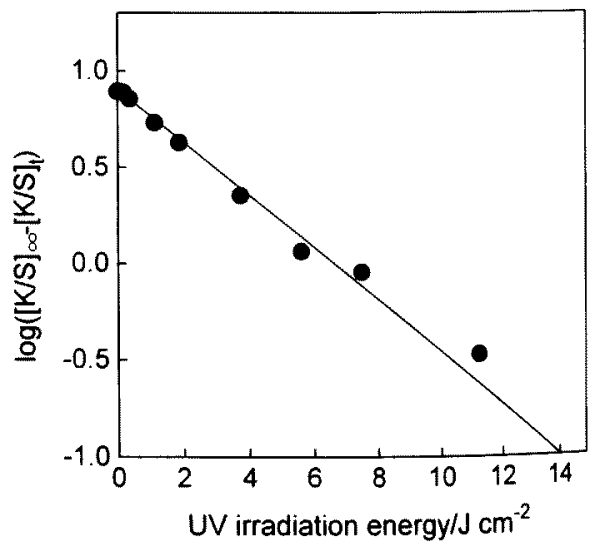

Fig. 3 Empirical linear relationship with log. arithmic equation taking plots of $\log \left[(K / S)_{\infty}-(K / S)_{t}\right]$ of the sample against the UV irradiation energy. 
and with definite energy, respectively.

This result indicates that the conversion of hydroxyl group in the cellulosic substrate to aldehyde group by the UV photooxidation follows the apparent first order reaction kinetics in the initial step.

Thus, photo-oxidized cellulose shows reducing ability for aqueous silver ion forming dark metal silver micro-deposit, and deep brown staining could be obtained with a certain kinetic function to the irradiation energy. Utilizing this reaction, patterned dark staining with the metal silver deposit could be obtained if one could make patterned UV irradiation on the cellulosic substrate followed by the deposition of metal silver with the reduction reaction from the aqueous silver ion solution. Fig. 4 shows an example of the patterned staining using patterned UV irradiation with a patterned mask.

This work was partially supported by the Grant of International Co-operation Program from the Ministry of Education, Science and Culture of Japan.

\section{References}

1. C. Kujirai, Sen'i Gakkaishi, 21, 183 (1965).

2. C. Kujirai, Sen'i Gakkaishi, 21, 260 (1965).

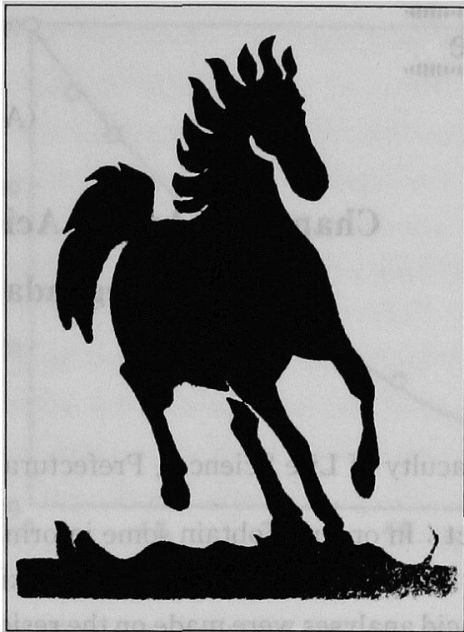

Fig. 4 Example of the patterned staining using patterned UV irradiation.

3. W. Harrison, J. Soc. Dyers Colour., 28, 359 (1912).

4. C. Kujirai, Sen'i Gakkaishi, 22, 84 (1966).

5. S. Matsumura, S.Maeda, and S.Yoshikawa, Makromol. Chem., 191, 1269 (1990).

6. M. Ueda, H-s. Shin, Y-s. Moon, and S. M Burkinshaw, J. Soc. Dyers Colour., submitted. 